\title{
Recrutamento de animais sésseis e sedentários em substrato artificial na área portuária do Recife - PE, com enfoque para a bioinvasão.
}

\section{Recruitment of sessile and sedentary animals on artificial substrate in the port area of Recife - PE, with focus on bioinvasion}

\author{
DOI: $10.46814 / \operatorname{lajdv3n4-040}$
}

Recebimento dos originais: 01/05/2021

Aceitação para publicação: 31/06/2021

\author{
Arthur V. O. M. Melo \\ Secretaria de Educação do Estado de Pernambuco. Recife, Brasil. Mestre em Biologia Animal - \\ UFPE \\ E-mail - arthur_marrocos@yahoo.com.br
}

Ralf Schwamborn

Depto. de Zoologia, Universidade Federal de Pernambuco (UFPE). Recife, Brasil.

\section{Cristiane M. R. Farrapeira}

Depto. de Biologia, Universidade Federal Rural de Pernambuco (UFRPE). Recife, Brasil.

\begin{abstract}
RESUMO
O Porto do Recife recebe uma grande quantidade de navios de vários tipos e de muitas partes do mundo, podendo trazer espécies exóticas incrustadas no casco ou em água de lastro. O objetivo do presente estudo foi registrar e analisar os padrões de recrutamento de espécies bentônicas sésseis, assim como investigar uma possivel introdução de espécies bentônicas exóticas e criptogênicas em uma área portuária com grande fluxo de navios. Utilizaram-se placas de metal galvanizado para estudo do recrutamento de animais sésseis e sedentários em três pontos amostrais no porto, com três réplicas cada, totalizando nove pontos, durante seis meses (outubro de 2010 a março de 2011), considerado como período seco. As placas de recrutamento foram retiradas a cada 30 dias durante seis meses. As principais espécies de acordo com a área de cobertura foram Mytella charruana (72\%) e Amphibalanus improvisus $(22 \%)$ e espaços vazios (7,5\%). Não houve diferença significativa quando comparadas as três estações no porto do Recife, sendo encontradas diferenças quando comparadas os dados das áreas de cobertura das espécies mais abundantes no recrutamento. Foram elas: Clytia gracilis, Obelia dichotoma, M. charruana e A. improvisus.
\end{abstract}

Palavras chaves: Animais Bentônicos, predação, espécies exóticas, sucessão ecológica.

\section{ABSTRACT}

The Port of Recife receives a large number of vessels of various types and from many parts of the world, and can bring exotic species encrusted hull or in ballast water. The aim of this study was to record and analyze the patterns of recruitment of sessile benthic species, as well as investigating a possible introduction of exotic and cryptogenic benthic species in a port area with great flow vessels. We used galvanized metal plates to study the recruitment of sessile and sedentary animals at three stations in the harbor, with three replicas each, totaling nine points, six months (October 2010 to March 2011), considered the dry. The plates recruitment were taken every 30 days for six months. The main species according to coverage area were Mytella charruana (72\%) and Amphibalanus improvisus $(22 \%)$ and voids $(7.5 \%)$. There was no significant difference when comparing the three stations in the 
Port of Recife, differences were found when comparing the coverage areas of most abundant species in recruitment. They were: Clytia gracilis, Obelia dichotoma, M. charruana and A. improvisus.

Keywords: benthic animals, predation, alien species, ecological succession.

\section{INTRODUÇÃO}

O recrutamento é a ocupação inicial de um substrato por organismos incrustantes "fouling" (TOMMASI et al., 1972; BAKER et al., 2004), que sobrevivem durante certo período de tempo após o assentamento (RodRiguez et al., 1993; BOOTH \& BROSNAM, 1995). Em uma placa experimental submersa, desenvolvem-se microrganismos após alguns minutos, formando um biofilme ou microincrustação (CALLOW \& CALLOW, 2002). Logo após esta fase, larvas de vários organismos bentônicos começam a recrutar (WHOI, 1952; OTSUKA \& DAUER, 1982), ocorrendo sucessão ecológica (ODUM, 1988).

De acordo com SUTHERLAND (1974) quatro semanas seria um período suficiente para estudar o recrutamento sobre substratos submersos, Nery et al. (2008) destacaram que cinco meses são suficiente para descrever a sucessão ecológica na Bacia Portuária do Recife.

Num determinado ambiente as espécies podem ser consideradas nativas, criptogênicas ou exóticas (ENO et al., 1977). CARLTON (2009) definiu como nativas as espécies ocorrentes naturalmente em um ambiente, com registro paleontológico; como exóticas, as observadas fora de seu limite de distribuição historicamente conhecido, resultado de dispersão acidental ou transferência intencional por atividades humanas; e como criptogênicas, aquelas cuja origem não é facilmente demonstrada e possuem ampla distribuição geográfica em baías, portos e estuários do mundo.

Nenhum habitat está imune à introdução de espécies exóticas, mas comumente aquelas que se tornam invasoras ocorrem em portos e marinas e circunvizinhanças (GOULLETQUER et al., 2002; COHEN et al., 2005; RAMADAN et al., 2006; FARRAPEIRA et al., 2009; RochA et al., 2012). As atividades de navegação constituem um dos principais meios de introdução de espécies em novas áreas (LEWIS et al., 2003), fato que vem aumentando devido ao aumento do comércio internacional (FOFONOFF et al., 2003; CARLTON, 2009; FARRAPEIRA et al., 2011). Estudos de colonização de invertebrados permitem conhecer a fauna local, assim como as mudanças ocorrentes na comunidade ao longo do tempo (CARVALHO \& UIEDA, 2004).

Estudos com placas de recrutamento têm sido realizados com o intuito de observar os processos de colonização e sucessão ecológica, e a introdução de espécies exóticas, em várias partes do mundo (CoE, 1932; Pech et al., 2002; RAMAdAn et al., 2006; Freestone et al., 2009). No Brasil, vários 
autores demonstraram a técnica como bom instrumento de gestão e diagnóstico ambiental, ao definir composição faunística e introdução de exóticas (MAYER-PINTO et al., 2000, SILVA et al., 2001, BREVES-RAMOs et al., 2005, FERNANDES et al., 2006, NEVES et al., 2007, XAVIER et al., 2008, NERY et al., 2008, CANGUSSU et al., 2010).

No Porto de Recife, por receber muitas embarcações de outros países (Farrapeira et al., 2007), faz-se necessário entender os processos de recrutamento e sucessão dos animais bentônicos, e monitorar a introdução de espécies exóticas no litoral pernambucano. Assim, o objetivo do presente estudo foi investigar uma possível introdução e estabelecimento de espécies bentônicas exóticas e/ou criptogênicas em uma área portuária com grande fluxo de navios no Porto do Recife. Buscou-se registrar e analisar os padrões de recrutamento e a dinâmica de sucessão de espécies bentônicas sésseis.

\section{MATERIAIS E MÉTODOS}

O trabalho foi realizado no Porto do Recife $\left(8^{\circ} 03^{\prime} \mathrm{S}\right.$ e $\left.34^{\circ} 51^{\prime} \mathrm{W}\right)$ no Estado de Pernambuco. A bacia portuária é formada pela desembocadura do rio Capibaribe e dos rios componentes da Bacia do Pina (rios Tejipió, Jiquiá, Pina e Jordão (FARRAPEIRA, 2006). O porto apresenta grande impacto causado pelo número de atracamento de embarcações (exportação, importação e transatlânticos) e, recebe uma média anual de 491 atracações de navios de (FARRAPEIRA et al., 2007).

O local, de acordo com FARRAPEIRA et al. (2010), funciona também como um reservatório de descarga de indústria e esgoto doméstico, com coliformes e bactérias termo-tolerantes presentes na água. $\mathrm{O}$ ambiente é considerado como um complexo estuarino-fluvial, com grande aporte de água doce (CPRH, 2001). A salinidade varia com o aumento da pluviometria no local, de cinco a 22, durante as marés baixas nos meses chuvosos, quando ocorre um forte aporte de água doce, enquanto que na estação seca alcança valores de 32, chegando ao máximo de 37 em preamares (SOMERFIELD et al., 2003; NERY et al., 2008; FARRAPEIRA et al., 2009). Por conta da grande sedimentação que ocorre na área, FERNANDES et al. (2006) observaram que a transparência da água atinge apenas uma profundidade média de $0,66 \mathrm{~cm}$.

A área apresenta um clima do tipo As' denominado Tropical Quente Úmido, segundo a classificação de Köppen, com uma média anual de temperatura de $25^{\circ} \mathrm{C}$ e da água variando entre 24 a $32^{\circ} \mathrm{C}$, e uma precipitação média de $1.763 \mathrm{~mm}$, dos quais, $80 \%$ caem durante o período de março a agosto (inverno) (SOMERFIELD et al., 2003). 


\section{CARACTERIZAÇÃO DO EXPERIMENTO}

O período de estudo foi de 30 de setembro de 2010 a 27 de março de 2011, totalizando seis meses. O paredão de atracagem do porto foi dividido em três pontos: estação I (Terminal Açucareiro), II (Terminal Graneleiro) e III (Cais inativo) (Fig. 1). A estação I é o cais de atracagem dos navios açucareiros e sofre maior influência marinha e dos estuários dos rios Capibaribe e Beberibe. A estação II localiza-se na região mediana da Bacia Portuária, sendo a área de maior atividade de atracagem de navios de grãos e transatlânticos. A estação III é conhecida como Marco Zero e fica na parte mais interna do porto, onde não ocorreram atividades portuárias durante o período do experimento. Em cada estação três equipamentos foram instalados de forma equidistante, a 100 metros um do outro, como réplicas nas suas respectivas estações.

Figura 1. Vista aérea da Bacia Portuária de Recife- PE, sinalizando as áreas de instalação dos experimentos. Estação I, local de atracagem dos navios açucareiros. Estação II, local de atracagem de navios graneleiros. Estação III, sem atividades portuárias.

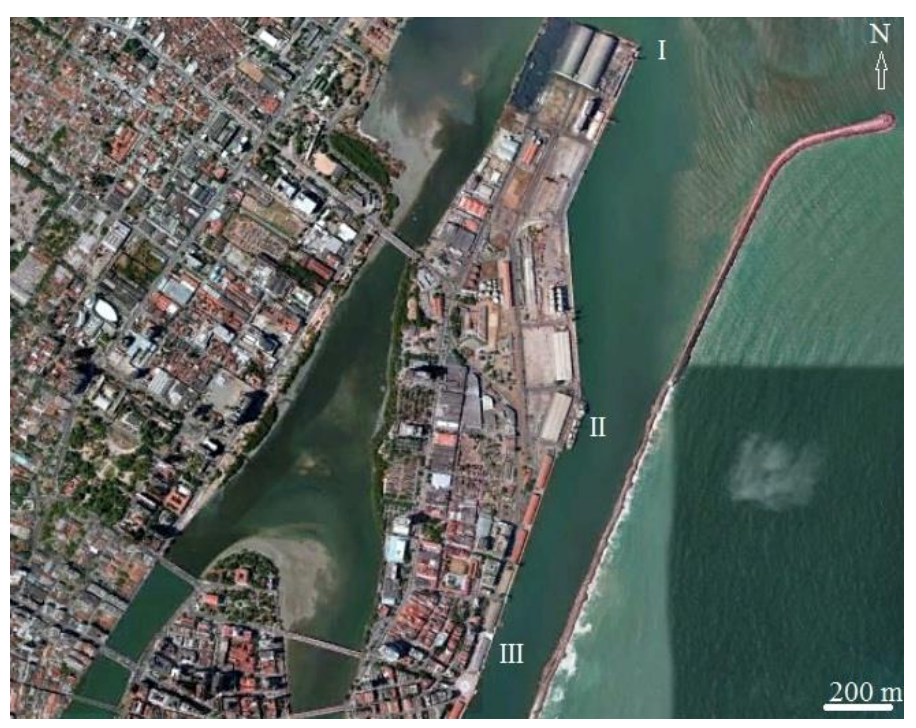

Fonte: Google earth.

Foi realizado um experimento para retratar a dinâmica de recrutamento dos animais sésseis. Para estudar o recrutamento foi removida uma placa de cada equipamento, a cada 30 dias, para análise do recrutamento ocorrido neste período (SUTHERLAND, 1974). Após a remoção, outra placa limpa era instalada em substituição, a fim de que esta representasse o recrutamento do mês subsequente. Este procedimento foi repetido até o fim do período de estudo.

Cada equipamento era formado por uma base de vergalhão de ferro e continham seis placas de metal galvanizado com $20 \mathrm{~cm}$ de comprimento por $10 \mathrm{~cm}$ de largura e $0,5 \mathrm{~cm}$ de espessura,distando 20 $\mathrm{cm}$ entre si, totalizando 54 placas. A estrutura foi mantida presa às defensas do porto, a um metro do menor nível de maré, durante a baixa mar (SILVA et al. 2001; NERY et al., 2008). 
Os corpos de provas (placas) removidos foram acondicionados em sacos plásticos etiquetados, contendo água do local e posteriormente fixadas em formolaldeído a 4\%, para ulterior triagem e identificação. Para a leitura das placas, utilizou-se uma placa de leitura com $10 \mathrm{~cm}$ x $8 \mathrm{~cm}$, contendo 80 quadrículas e interseções de 1 x $1 \mathrm{~cm}$. A leitura das placas foi feita pelo método de contagem dos organismos presentes no ponto de interseção das quadrículas, considerando que este método é um dos mais eficientes no estudo de comunidades incrustantes (SILVA et al., 2001; MACEDO et al., 2006; NERY et al., 2008).

A área da placa que não se enquadrou na rede de quadrículas foi observada para identificação taxonômica dos invertebrados, sendo considerada como fauna associada, para melhor caracterização da biota, seguindo NERY et al. (2008).

Foram calculadas as áreas de cobertura e as frequências relativas das espécies em todos os meses. Segundo NERY et al. (2008), as espécies foram classificadas quanto à frequência, em: muito frequente $(>70 \%)$, frequente $(30 \%$ a $70 \%)$, pouco frequente $(10 \%$ a $30 \%)$ e raras $(<10 \%)$.

A classificação das espécies quanto ao status de distribuição geográfica e de origem seguiu a definição de FARRAPEIRA et al. (2011), considerando espécies nativas aquelas com uma longa presença na área geográfica estudada; as exóticas, as que foram trazidas de outras regiões, por apresentarem descontinuidade geográfica grande entre a região onde foram originalmente descritas como nativas e a área onde foram encontradas na costa brasileira, e criptogênicas, aquelas em que não havia nenhum tipo de informação sobre sua localidade de origem ou que não foram classificadas como nativas ou exóticas de nenhuma região específica.

As abundâncias de cada estação foram comparadas usando a ANOVA Kruskal-Wallis, usando o software BioEstat 5.0. Foi gerada uma matriz de similaridade de Bray-Curtis sobre o $\log (\mathrm{x}+1) \mathrm{da}$ abundância dos animais em recrutamento e a sucessão mais recente das abundâncias das populações mais abundantes em cada equipamento. A partir desta matriz foi feita uma ANOSIM (CLARKE \& WARWICK, 2001) usando o software Primer 6.0. Os dados pluviométricos foram obtidos do Instituto Nacional de Meteorologia - Inmet.

\section{RESULTADOS}

Os experimentos foram eficientes por registrarem as dinâmicas de recrutamento e sucessão nas condições ambientais do Porto do Recife. Espécies exóticas, criptogênicas e nativas foram registradas, sobrevivendo aos impactos locais.

A preciptação variou de 23,5 a 329,5 mm (Fig. 2), destacando-se que no período de dezembro de 2010 a fevereiro de 2011 houve mais chuvas do que a média histórica registrada para o período. 
Fig 2. Pluviosidade média mensal do Porto do Recife, Pernambuco, durante os meses de outubro de 2010 a março de 2011. INMET.

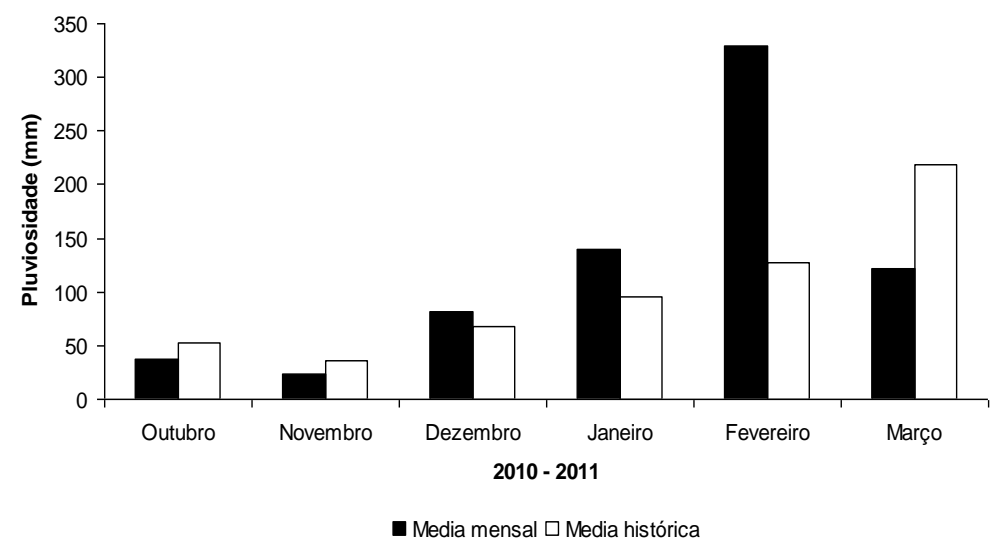

\section{EXPERIMENTOS DE RECRUTAMENTO}

Nos corpos de provas (placas) foram encontrados 30 táxons pertencentes a seis filos animais, dentre os quais foram identificadas 16 espécies, os demais, pertencentes ao Filo Polychaeta, identificados no nível de Família (Tab. I). Foram perdidas três placas, uma no mês de fevereiro (estação I), duas no mês de março (estação III).

Com relação à fauna associada, além das espécies já mencionadas (Tab. I), foram encontrados o mexilhão sedentário Mytilopsis leucophaeta Conrad, 1831 e a fauna vágil: planária Distylochus martae Marcus, 1947, gastrópodos Melanoides tuberculatus Müller, 1774, Nassarius vibex Say, 1822, Rissoina cancelata Philippi, 1847, poliquetas das famílias Ampharetidae, Cirratulidae, Dorvilleidae, Eunicidae, Hesionidae, Nereididae, Pholoididae, Phyllodocidae, Polynoidae, Syllidae e Terebellidae e pantópodo Anoplodactylus californicus Hall, 1912.

Foram encontradas 10 espécies não nativas do Brasil (cinco exóticas e cinco criptogênicas) e seis nativas. Foram mais frequentemente e recrutadoras iniciais as espécies criptogênicas: Clytia gracilis Sars, 1850, Obelia dichotoma Linnaeus, 1758, Amphibalanus improvisus Darwin, 1854, A. amphitrite Darwin, 1854 e Bowerbankia gracilis Leidy, 1855, porém sem regularidade mensal na frequência. Dentre as espécies exóticas duas merecem destaque pelas estratégias de ocupação espacial, em interstícios de cirrípedes e interior de testas vazias destes animais: anêmona Haliplanella lineata Verrill, 1869 e tanaidaceo Sinelobus stanfordi Richardson 1901. A espécie nativa Mytella charruana d'Orbigny, 1842 recrutou todos os meses, ocupando, na maioria das vezes, no sentido periferia das placas para o centro. As ostras Crasssostrea rhizophorae Guilding, 1828 recrutaram nos meses de janeiro a março de 2011 sendo encontrados apenas indivíduos jovens. 
Tabela 1. Frequência e área de cobertura das espécies encontradas nas placas de recrutamento no Porto do Recife, de outubro de 2010 a março de 2011, onde: status (ST) Status: C = criptogênica, E= exótica, N = nativa, "?"= status desconhecido; Int $=$ interseção, FAs = Fauna Associada (assinalada com +); F = Frequência em \%; AC = Área de Cobertura em \%.

\begin{tabular}{|c|c|c|c|c|c|}
\hline TAXON & ST & Int & FAs & $\mathbf{F}$ & $\mathbf{A C}$ \\
\hline \multicolumn{6}{|l|}{ CNIDARIA } \\
\hline Haliplanella lineata & $\mathrm{E}$ & $\mathrm{X}$ & $\mathrm{X}$ & 13.7 & 1.1 \\
\hline Clytia gracilis & $\mathrm{C}$ & $\mathrm{X}$ & $\mathrm{X}$ & 56.9 & 19.3 \\
\hline Obelia dichotoma & $\mathrm{C}$ & $\mathrm{X}$ & $\mathrm{X}$ & 55.4 & 12.2 \\
\hline \multicolumn{6}{|l|}{ PLATYHELMINTHES } \\
\hline Distylochus martae & $\mathrm{N}$ & & $\mathrm{X}$ & - & - \\
\hline \multicolumn{6}{|l|}{ MOLLUSCA } \\
\hline Crassostrea rhizophorae & $\mathrm{N}$ & $\mathrm{X}$ & $\mathrm{X}$ & 15.7 & 1.4 \\
\hline Mytella charruana & $\mathrm{N}$ & $\mathrm{X}$ & $\mathrm{X}$ & 64.7 & 9.8 \\
\hline Mytilopsis leucophaeta & $\mathrm{E}$ & - & $\mathrm{X}$ & - & \\
\hline Melanoides tuberculatus & $\mathrm{E}$ & - & $\mathrm{X}$ & - & \\
\hline Nassarius vibex & $\mathrm{N}$ & - & $\mathrm{X}$ & - & \\
\hline Rissoina cancelata & $\mathrm{N}$ & - & $\mathrm{x}$ & & \\
\hline \multicolumn{6}{|l|}{ ANNELIDA } \\
\hline Ampharetidae & $?$ & - & $\mathrm{X}$ & - & \\
\hline Cirratulidae & $?$ & - & $\mathrm{X}$ & - & \\
\hline Dorvilleidae & $?$ & - & $\mathrm{x}$ & - & \\
\hline Eunicidae & $?$ & - & $\mathrm{X}$ & + & \\
\hline Hesionidae & $?$ & - & $\mathrm{X}$ & + & \\
\hline Nereididae & $?$ & - & $\mathrm{X}$ & + & \\
\hline Pholoididae & $?$ & - & $\mathrm{X}$ & - & \\
\hline Phyllodocidae & $?$ & - & $\mathrm{x}$ & - & \\
\hline Polynoidae & $?$ & - & $\mathrm{x}$ & - & \\
\hline Sabellidae & $?$ & $\mathrm{X}$ & $\mathrm{x}$ & 23.2 & 2.9 \\
\hline Serpulidae & $?$ & $\mathrm{X}$ & $\mathrm{X}$ & - & \\
\hline Spionidae & $?$ & $\mathrm{X}$ & $\mathrm{x}$ & 25.5 & 1.7 \\
\hline Syllidae & $?$ & - & $\mathrm{X}$ & - & \\
\hline Terebellidae & $?$ & - & $\mathrm{X}$ & - & \\
\hline \multicolumn{6}{|l|}{ ARTHROPODA } \\
\hline \multicolumn{6}{|l|}{ PYCNOGONIDA } \\
\hline Anoplodactylus californicus & $\mathrm{E}$ & - & $\mathrm{x}$ & - & \\
\hline \multicolumn{6}{|l|}{ CIRRIPEDIA } \\
\hline Amphibalanus amphitrite & $\mathrm{C}$ & $\mathrm{X}$ & $\mathrm{X}$ & 11.8 & 1.2 \\
\hline Amphibalanus eburneus & $\mathrm{N}$ & $\mathrm{X}$ & $\mathrm{X}$ & 2.0 & 1.0 \\
\hline Amphibalanus improvisus & $\mathrm{C}$ & $\mathrm{X}$ & $\mathrm{X}$ & 88.2 & 39.5 \\
\hline \multicolumn{6}{|l|}{ TANAIDACEA } \\
\hline Sinelobus stanfordi & $\mathrm{E}$ & $\mathrm{X}$ & $\mathrm{x}$ & 5.9 & 1.5 \\
\hline \multicolumn{6}{|l|}{ BRYOZOA } \\
\hline Bowerbankia gracilis & $\mathrm{C}$ & $\mathrm{X}$ & $\mathrm{X}$ & - & \\
\hline
\end{tabular}


Não houve diferença significativa entre as abundâncias dos recrutamentos, indicando que o recrutamento na Bacia Portuária do Recife é similar em todas as estações. A Anosim feita entre as estações a partir das espécies mais abundantes indicou que não existe diferença significativa na composição das espécies entre as estações. Já na análise entre as espécies por mês, foram encontradas diferenças significantes, A. improvisus entre os meses 1 e 6; 2 e 3; 2 e 4; 2 e $5 ; 2$ e 6, os hidróides entre os meses 1 e $3 ; 2$ e 3; 2 e 4, os espaços vazios entre os meses 3 e 4 (Tab. II).

Tabela II. Anosim das espécies mais abundantes entre os meses de outubro de 2010 a março de 2011 no Porto do Recife PE. Onde $(\mathrm{R})=$ Recrutamento, $(-)=$ sem registro.

\begin{tabular}{ccc}
\hline Espécie & $\mathrm{H}(\mathrm{R})$ & $\mathrm{p}(\mathrm{R})$ \\
\hline Clytia gracilis & 23,18 & 0,01 \\
Obelia dichotoma & 23,14 & 0,01 \\
Mytella charruana & 7,16 & 0,02 \\
Polychaeta & - & - \\
Amphibalanus improvisus & 27,46 & 0,01 \\
Espaço vazio & 13,67 & 0,02 \\
\hline
\end{tabular}

Verificou-se uma maior presença de A. improvisus, O. dichotoma, C. gracilis e M. charruana nas estações I e II; na Estação II, registrou-se também a ocorrência de A. amphitrite e espaços vazios. Placas de todos os meses amostrados findaram seu período de exposição com espaços vazios, sobretudo nas estações II e III.

Durante os meses de recrutamento a espécie A. improvisus foi a que mais recrutou nos dois primeiros meses, tendo $O$. dichotoma e $C$. gracilis aumentado sua área de cobertura principalmente por causa do alto índice pluviométrico de dezembro a fevereiro de 2011. O mesmo aconteceu com os espaços vazios e o recrutamento de $M$. charruana, que também teve sua população influenciada pelas chuvas (Fig. 3).

Fig. 3. Comparação mês a mês das espécies mais frequentes na dinâmica do recrutamento durante os meses de outubro de 2010 a março de 2011 no Porto do Recife - PE. Ai = Amphibalanus improvisus; Hi = hidróides (Obelia dichotoma, Clytia gracilis); My = Mytella charruana $; \mathrm{Vz}=$ Vazio; Outros = Haliplanella lineata, Crassostrea rhizophorae, A. amphitrite, A. eburneus, polychaetas, Sinelobus stanfordi, Bowerbankia gracilis.

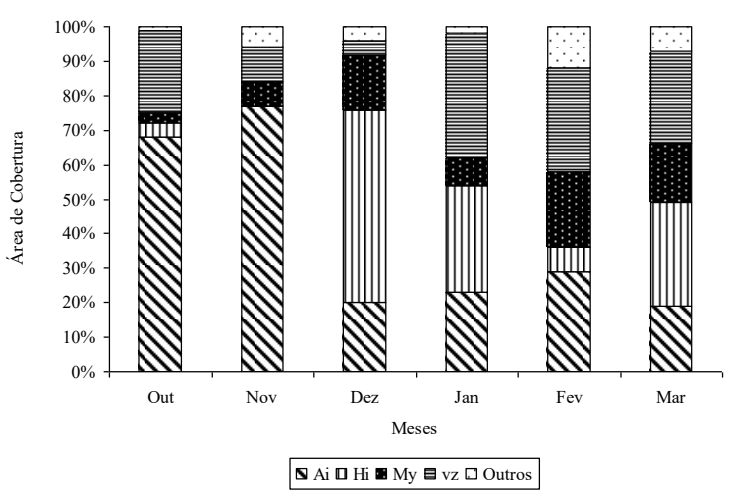


Nas placas de recrutamento foi observada a mortalidade de diversos espécimes de cirrípedes, sobretudo nas placas onde havia planárias Distylochus martae. No mês de novembro, dos 552 indivíduos de A. improvisus fixados, 123 estavam mortos, enquanto se encontraram 43 planárias. Fato semelhante foi observado em relação às colônias de hidróides $C$. gracilis e $O$. dichotoma e a presença de Anoplodactylus californicus. Nas placas em que este conjunto apareceu havia muitos espaços vazios e colônias predadas.

\section{DISCUSSÃO}

O presente estudo encontrou no recrutamento 16 espécies, sendo dessas 11 espécies não nativas do Brasil, as mais frequentes foram as criptogênicas $C$. gracilis e A. improvisus e a exótica $O$. dichotoma, demonstrando o sucesso dessas espécies nesse processo.

\subsection{DISPERSÃO DOS ORGANISMOS}

CARRIKER (1992) afirmou que a dispersão geográfica bem sucedida de uma espécie é produto de interação entre propriedades fisiológicas do organismo e da qualidade ambiental. Para PETERSON $e t$ al. (2003) embora os fatores bióticos sejam importantes em tornar o ambiente mais suscetível à invasão, os fatores abióticos servem como "filtro" para as introduções e limitam o estabelecimento de espécies exóticas ou criptogênicas a condições ambientais similares às das suas regiões originais.

Os vetores de dispersão apontados como responsáveis pela introdução inadvertida de muitos organismos bentônicos nas águas costeiras de todos os oceanos em regiões litorâneas são, principalmente, a bioincrustação de animais nos cascos das embarcações ou na água de lastro (SKeRman, 1960; Williams et al., 1988; Ruiz et al., 2000a; 2000b; Gollasch, 2002; FofONOFF et al., 2003; COUTTS \& TAYLOR, 2004; FARRAPEIRA et al., 2007; DAVIDSON et al., 2008; FARRAPEIRA et al., 2010; 2011), como ocorreu na área de estudo com H. lineata, O. dichotoma, M. leucophaeta e B. gracilis (FARRAPEIRA et al. 2011).

\subsection{EVENTOS ABIÓTICOS}

O período estudado é classificado como verão, pelo comportamento climático histórico (SOMERFIELD et al., 2003), mas se apresentou particularmente chuvoso nos meses de dezembro de 2010 a fevereiro de 2011. Assim, a semelhança encontrada tanto no recrutamento quanto na sucessão para todos os meses indica que não houve influência da pluviosidade e a consequente diminuição de salinidade local sobre os animais. MUNIZ et al. (2005) verificaram que a circulação das correntes na bacia portuária causam a mistura completa das águas e deixa o ambiente homogêneo, o que também 
dá suporte aos resultados. Todos os táxons identificados são reconhecidamente estuarinos ou, pelo menos, eurihalinos (FARRAPEIRA et al., 2011).

Segundo Glasby e CONNELl (2001), as larvas planctônicas de invertebrados marinhos bentônicos tendem a se fixar tão logo encontrem um substrato natural ou artificial adequado, que facilite sua colonização. Porém, segundo KENNISH (1994), os impactos ambientais associados a um processo de intensa movimentação de sedimentos podem provocar efeitos tanto no habitat quanto nos organismos, alterando a qualidade da água, relocando os sedimentos, nesta dinâmica, aumentando a mortalidade dos organismos devido à ressuspensão do sedimento de fundo e afetando, sobretudo os animais filtradores. Essas condições foram relevantes para a dinâmica ecológica observada na área em algumas estações, sobretudo na Estação II (Terminal Graneleiro), que, coincidentemente era a estação de atracagem dos navios transatlânticos.

Para XAVIER et al. (2008) um distúrbio físico no ambiente abre novos espaços para colonização e pode interferir nos processos de competição por uma determinada fonte, sendo uma causa importante da heterogeneidade da estrutura e dinâmica natural da comunidade, não apenas eliminando uma parte da cobertura da comunidade, aumentando a quantidade de nutrientes disponíveis, e a morte dos indivíduos. A ressuspensão de sedimentos motivada, tanto pela atracagem de navios transatlânticos (Estação II), quanto pelo aporte maior de sedimentos nos meses mais chuvosos afetou igualmente a dinâmica observada no recrutamento

Em janeiro de 2011 apenas seis espécies foram observadas fixadas nas placas, sendo considerado um dos meses como menor número de espécies; essa diminuição da diversidade estaria relacionado ao alto índice pluviométrico no período $(139,10 \mathrm{~mm})$, o que fugia ao padrão de chuvas esperado para o mês. Para KROHLING \& ZALMON (2008) as chuvas trazem mais material particulado em suspensão e sedimentação inorgânica, o que prejudica a colonização. Porém, favorece a aparição de espécies oportunistas, conforme destacado por FLYNN \& VALERIO-BERARDO (2012), que relataram a rápida colononização de substrato artifical pelos hidróides $O$. dichotoma e $C$ gracilis, sendo essas espécies consideradas as mais abudantes no período onde ocorrem altos índices de chuvas.

\subsection{DINÂMICA DO RECRUTAMENTO}

O fato de terem sido contabilizados 30 táxons nas placas de recrutamento mostra, entretanto, que a área estudada, apresenta um aumento no número de espécies no infralitoral quando comparado com NeRY et al. (2008). Por outro lado, apesar da bacia portuária ser qualificada como um ambiente poluído, o número de taxa excede o encontrado por BREVES-RAMOS et al. (2005), em placas 
experimentais na Baía de Guanabara, Rio de Janeiro, e se equipara ao encontrado por EMARA \& BELAL (2004), no Canal de Suez, ambientes igualmente poluídos.

Nesse estudo, o cirrípede criptogênico A. improvisus foi a única espécie considerada mais frequente, em todos os meses pesquisados. CANGUSSU et al. (2010) destacaram a sua grande quantidade em placas experimentais, classificando-o como uma das primeiras espécies a recrutarem. Esta espécie compreende mais de $90 \%$ da população bentônica fixada em superfícies artificiais (WEISS, 1947), sendo já relatada como principal recrutadora em placas experimentais em diversas regiões (MAYERPINTO et al., 2000; BERNTSSON \& JONSSON, 2003; SluYs et al., 2005). FARRAPEIRA et al. (2007) destacam que esta espécie foi a única presente em quase todas as embarcações que acostavam no Porto do Recife. FARRAPEIRA et al. (2009) a relataram na faixa inferior do mediolitoral fixada diretamente em substratos naturais e artificiais e sobre outros organismos na Baía de Suape, Pernambuco, e este padrão de fixação foi observado por FARRAPEIRA (2006) e FARRAPEIRA et al. (2009) em toda a área estuarina do Recife, sendo dominante na faixa inferior dos médio e infralitoral, semelhantemente ao que ocorreu neste trabalho. A alta porcentagem de animais mortos pode ser explicada pela presença da planária D. martae. As planárias são reconhecidamente predadores de cirrípedes (HURLEY, 1976; SLUYS et al., 2005; LEE et al., 2006).

As demais espécies de cirrípedes encontradas, a criptogênica $A$. amphitrite e a nativa $A$. eburneus não estiveram bem representadas numericamente nas placas estudadas. Amphibalanus amphitrite é uma das espécies mais estudadas do "fouling" (EDMONDSON \& INGRAM, 1939; GHOBASHY \& El-Komy, 1981; MAYer-Pinto \& Junqueira, 2003; Zvyagintsev, 2003; Floerl et al., 2004; RAMADAN et al., 2006; FREESTONE et al., 2009). Na área de estudo, porém, esta espécie é encontrada preferencialmente na zona média do mediolitoral (FARRAPEIRA, 2006), não contemplada neste estudo. Embora A. eburneus também seja igualmente encontrada em placas (GHOBASHY \& EL-KOMY, 1981; MAYER-PINTO et al., 2000; RAMADAN et al., 2006), ela é incapaz de se fixar em superfícies artificiais de metal (WEISS, 1947), apesar de ser uma das espécies mais adaptadas e resistentes a águas poluídas (RELINI, 2003). Na área de estudo, FARRAPEIRA (2006) e FARRAPEIRA et al. (2009), encontrou-a apenas nas áreas inferiores do mediolitoral e infralitoral, epibiôntica em conchas de bivalves.

Nas placas de recrutamento duas espécies de hidróides: $C$. gracilis e $O$. dichotoma, foram registradas, com frequência variável em relação aos meses de estudo. A presença do hidróide criptogênico $O$. dichotoma em placas experimentais tem sido relatada com frequência (NANDAKUMAR, 1995; MAYER-PINTO et al., 2000; RELINI et al., 2000; BREVES-RAMOS et al., 2005; FLYNN \& VALERIOBERARDO, 2012). Esta espécie é reportada como tolerante em locais com índices elevados de eutrofização e baixa salinidade (BREVES-RAMOS et al., 2005). Já o exótico C. gracilis, ocasionalmente 
observado em placas (Migotto, 1996; CANGussu et al., 2010; Flynn \& VAlERIO-Berardo, 2012), é comumente mencionado como epizóica em vários invertebrados bentônicos (MIGOTTO, 1996), fato também observado durante este experimento. Foram classificadas como muito frequentes, nas placas de recrutamento, especialmente nos meses chuvosos, e, posteriormente, apenas como epibiontes na sucessão, corroborando as observações de MigotTo (1996) e MigotTo et al. (2001).

\section{CONCLUSÕES}

Foram encontradas mais espécies exóticas e criptogênicas do que nativas $\operatorname{tant\ominus ~no~preocesso~de~}$ recrutamento quanto no de sucessão, o estabelecimento das espécies exóticas Haliplanella lineata e Sinelobus stanford e o aumento das chuvas aumentaram o recrutamento dos hidróides Clytia gracilis e Obelia dichotoma, a presença das espécies Dystilocus martae e Anoplodactylus californicus controlaram a presença de Amphibalanus improvisus e dos hidróides C. gracilis e O. dichotoma respectivamente. A. eburneus foi encontrada em maior quantidade nas placas de sucessão, as espécies exóticas Mytilopsis leucophaeta e Melanoides tuberculatus só foram encontradas em placas de sucessão. A biota portuária não diferiu nos três pontos demonstrando uma uniformidade da fauna para o local.

\section{AGRADECIMENTOS}

À Fundação CAPES, pela bolsa do mestrado e à administração do Porto do Recife S.A., pela autorização para o desenvolvimento do trabalho na área. 


\section{REFERÊNCIAS BIBLIOGRÁFICAS}

BAKER, P.; BAKER, S. M. \& FAJANS, J. 2004. Nonindigenous marine species in the greater. TAMPA BAY ECOSYSTEM. TAMPA BAy ESTUARY PROgRAM TECH. PUBL., N. 02-04

BERNTSSON, K. M. \& JONSSON, P. R. 2003. Temporal and spatial patterns in recruitment and succession of a temperate marine fouling assemblage: a comparison of static panels and boat hulls during the boating season. Biofouling 19(3): 187-195.

BoOTh, D. J. \& BRosnan, D. M. 1995. The role of recruitment dynamics in rocky shore and coral reef fi sh communities. Advances in Ecological Research 26: 309-385.

Breves-Ramos, A.; LAVRado, H. P.; JUnQUeIRA, A. O. R. \& Silva, S. H. G. 2005. Succession in rocky intertidal benthic communities in areas with different pollution levels at Guanabara Bay (RJ-Brazil). Brazilian Archives of Biology and Technology 48(6): 951-965.

Callow, M. E. \& CAllow, J. A. 2002. Marine biofouling sticky problem. Biologist 49(1): 1-5.

Cangussu, L. C.; Kremer, L. P.; Rocha, R. M.; Pitombo, F. B.; Heyse, H. \& Bornancin, E. C. 2010. Substrate type as a selective tool against colonization by non-native sessile invertebrates.

Brazilian Journal of Oceanography 58(3): 219-231.

Carlton, J. T. 2009. Deep invasion ecology and the assembly of communities in historical time. In: RILOV, G. \& CROOKS, J.A. eds. Biological invasions in marine ecosystems. Ecological Studies 204. Berlin, Springer-Verlag. p. 13-56.

CARRIKER, M.R. 1992. Introductions and transfers of molluscs: risk considerations and implications. Journal of Shellfish Research 11: 507-510.

Carvalho, E. M. \& UiEDA, V. S. Colonização por macroinvertebrados bentônicos em substrato artificial e natural em um riacho da serra de Itatinga, São Paulo, Brasil. Revista Brasileira de Zoologia, 21(2) 287-293. 2004.

CLARKE, K. R. \& WARWICK, R. M. 2001. Change in marine communities: an approach to statistical analysis and interpretation. Bournemouth, Bourne Press. $128 \mathrm{p}$.

COE WR. 1932. Sexual phases in the American oyster (Ostrea virginica). Biol Bull. 63:419-441.

Cohen, A. N.; Harris, L.; Bingham, B. L.; Carlton, J.; Chapman, J.; LAmbert, C. C.; Lambert, G.; Luubenkov, J. C.; MurRay, S. N.; RaO, L.C.; REARdOn, K. \& Schwindt, E. 2005. Rapid assessment survey for exotic organisms in southern California bays and harbors, and abundance in port and nonport areas. Biological Invasions 7(6): 995-1002.

COUTTS, A. D. M. \& TAYLOR, M. D. 2004. A preliminary investigation of biosecurity risks associated with biofouling on merchant vessels in New Zealand. New Zealand Journal of Marine and Freshwater Research 38(2): 215-229. 
CPRH. 2001. Relatório de monitoramento de bacias hidrográficas do Estado de Pernambuco. Recife: Companhia Pernambucana de Controle de Poluição Ambiental e de Administração dos Recursos Hídricos- CPRH. 110 p.

Davidson, I. C.; McCann, L. D.; Fofonoff, P. W.; Sytsma, M. D. \& RuIZ, G. M. 2008. The potential for hull-mediated species transfers by obsolete ships on their final voyages. Diversity and Distributions 14(3): 518-529.

Edmondson, C.H. \& IngRAM, W.M. 1939. Fouling organisms in Hawaii. Occasional Papers of the Bernice Pauahi Bishop Museum of Polynesian Ethnology and Natural History (14): 251-300. Emara, A. M. \& Belal, A. A. 2004. Marine fouling in Suez Canal, Egypt. Egyptian Journal of Aquatic Research 30(A): 189-206.

Eno, N.C.; Clark, R.A. \& SAnderson, W.G. (Eds.) 1997. Non-native marine species in British waters: a review and directory. Peterborough: Joint Nature Conservation Committee, $136 \mathrm{p}$.

FARRAPEIRA, C. M. R. 2006. Barnacles (Cirripedia Balanomorpha) of the estuarine region of Recife, Pernambuco, Brazil. Tropical Oceanography 34(2): 100-119.

Farrapeira, C. M. R.; Melo, A. V. O. M.; Barbosa, D. F. \& Silva, K. M. E. 2007. Ship hull fouling in the Port of Recife, Pernambuco. Brazilian Journal of Oceanography 55(3): 207-221.

Farrapeira, C. M. R.; Ramos, C. A. C.; Barbosa, D. F.; Melo, A. V. O. M.; Pinto, S. L.; VerÇOSA, M. M.; OliVEIRA, D. A. S. \& FRANCISCO, J. A. 2009. Zonación vertical de la macrofauna de sustratos sólidos del estuario del Río Massangana, Bahía de Suape - Pernambuco, Brasil. Biota Neotropica 9(1): 1-12.

Farrapeira, C. M. R.; Mendes, E. S.; Dourado, J. \& Guimarães, J. 2010. Coliforms accumulation in Amphibalanus amphitrite (Darwin, 1854) (Cirripedia) and its use as organic pollution bioindicator in the estuarine area of Recife, Pernambuco, Brazil. Brazilian Journal of Biology 70(2): 301-309.

FArRAPEira, C. M. R.; TenÓRIO, D. O. \& Amaral, F. M. D. 2011. Vessel biofouling as an inadvertent vector of benthic invertebrates occurring in Brazil. Marine Pollution Bulletin 62(3): 1538-1544.

Fernandes, M. L. B.; Paiva, R. J C.; Gonçalves, K. L. F.; SoAres, A. M. C.; Silva, M. L.; Silva. A. K. P. S. \& CHAVES, A. C. 2006.Viabilidade do suururu Mytella charruana (Mollusca: Bivalvia) como indicadora em estudos de monitoramento ambiental na região portuária do Recife - PE. Lumen 14(1): 101-103.

Floerl, O.; PoOl, T. K.; Inglis, G. J. 2004. Positive interactions between nonindigenous species facilitate transport by human vectors. Ecological Applications 14(6): 1724-1736.

FlynN, M. N. \& VALÉRIO-BERARDO, M. T. 2012. Avaliação da toxicidade in situ através do recrutamento de comunidade incrustante em painéis artificiais em terminal da Petrobrás, Canal de São Sebastião, São Paulo. Revista Intertox de Toxicologia, Risco Ambiental e Sociedade 5(1): 103-114.

Fofonoff, P. F.; Steves, B.; Miller, W. A.; Ruiz, G. \& Carlton, J. 2003. In ships or on ships? Unravelling the relative contribution of ballast tanks vs. hull fouling to ship-mediated invasions of 
North America by marine species. In: Third International Conference on Marine Bioinvasions. La Jolla, s. ed. p. 1- 42.

Freestone, A. L.; OSMAN, R. W. \& Whitlatch, R. B. 2009. Latitudinal gradients in recruitment and community dynamics in marine epifaunal communities: Implications for invasion success. In: LANG, M. A.; MacIntyre, I. G.; RÜtZler, K. eds. Proceedings of the Smithsonian Marine Science Symposium. Washington, D.C., Smithsonian Contributions to the Marine Sciences. n. 38. pp. $247-$ 258.

Ghobashy, A. F. A. \& El-Komy, M. M. 1981. Fouling in the southern region of the Suez Canal. Hydrobiological Bulletin 14(3): 179-185.

Glasby, T. M. \& ConNEL, S. D. 2001. Orientation and position of a substratum have large effects on epibiotic assemblages. Marine Ecology Progress Series 214: 127-135.

Gollasch, S. 2002. The importance of ship hull fouling as a vector of species introductions into the North Sea. Biofouling 18(2): 105-121.

GoulletQuer. P.; Bachelet, G.; Sauriau, P. G. \& Noel, P. 2002 Open Atlantic coast of Europe a century of introduced species into French waters. In: LEPPÄKOSKI, E. GOLLASCH, S.; OLENIN, S. (eds) Invasive aquatic species of Europe: distribution, impacts and management. Kluwer, Dordrecht, pp 276290

HuRLEY, A. C. 1976. The polycladid flatworm Stylochus tripartitus Hyman as a barnacle predator. Crustaceana 31(1): 110-111.

KenNiSH, M. J. 1994. Practical handbook of marine science. New York, John Wiley \& Sons, Inc. $562 \mathrm{p}$.

KROHLing, W. \& ZaLmon, I. R. 2008. Epibenthic colonization on an artificial reef in a stressed environment off the north coast of the Rio de Janeiro State, Brazil. Brazilian Archives of Biology and Technology 51(1): 213-221.

LeE, K.-M.; BeAl, M. A. \& Johnston, E.L. 2006. A new predatory flatworm (Platyhelminthes, Polycladida) from Botany Bay, New South Wales, Australia. Journal of Natural History 39(47): 3987-3995.

Lewis, P. N.; HewitT, C. L.; Riddle, M. \& McMinn, A. 2003. Marine introductions in the Southern Ocean: an unrecognised hazard to biodiversity. Marine Pollution Bulletin 46(2): 213-223.

MACEDO, I. M.; MASI, B. P. \& ZALMON, I. R. 2006. Comparison of rocky intertidal community sampling methods at the northern coast of Rio de Janeiro State, Brazil. Brazilian Journal of Oceanography 54(2/3): 147-154.

MAYER-PINTO, M. \& JUNQUEIRA, A. O. R. 2003. Effects of organic pollution on the initial development of fouling communities in a tropical bay, Brazil. Marine Pollution Bulletin 46(11): 1495-1503.

Mayer-Pinto, M.; Viana, M. S.; Lavrado, H. P.; Silva, T. A. \& Silva, S. H. G. 2000. Epibiosis on barnacles at Angra dos Reis. RJ: eutrophication effects. Nauplius 8(1): 55-61 
Migotto, A. E. 1996. Benthic shallow-water hydroids (Cnidaria, Hydrozoa) of the coast of São Sebastião, Brazil, including a checklist of Brazilian hydroids. Zoologische Verhandelingen 306: 1125.

Muniz, P.; Venturini, N.; Pires-Vanin, A. M. S.; Tommasi, L. R. \& BorJa, A. 2005. Testing the applicability of Marine Index (AMBI) to assessing the ecological quality of soft-bottom benthic communities, in the South America Region. Marine Pollution Bulletin 50: 524-637.

NANDAKUMAR, K. 1995. Competitive interactions among sessile organisms in Tomioka Bay, south Japan: importance of light conditions on the panel surface. Marine Biology 121(4): 713-719

Nery, P. P. C. F.; Leitão, S. N.; Fernandes, M. L. B.; Silva, A. K. P. \& Chaves, A. C. 2008. Recrutamento e sucessão ecológica da macrofauna incrustante em substratos no Porto do Recife - PE, BRASIL. Revista Brasileira de Engenharia de Pesca 3(1): 51-61.

Neves, C. S.; Rocha, R. M.; Pitombo, F. B. \& Roper, J. J. 2007. Use of artificial substrata by introduced and cryptogenic marine species in Paranaguá Bay, southern Brazil. Biofouling 23(5): 31930 .

ODUM, E. P. 1988. Ecologia. Rio de Janeiro: Editora Guanabara.

Otsuka, C. M. \& DAueR, D. M. 1982. Fouling community dynamics in Lynnhaven Bay, Virginia. Estuaries 5(1): 10-22.

PeCh, D.; ARdisson, P.-L. \& BouRget, E. 2002. Settlement of a tropical marine epibenthic assemblage on artificial panels: Influence of substratum heterogeneity and complexity scales. Estuarine, Coastal and Shelf Science 55: 743-750.

Peterson, A. T.; PAPES, M. 7 KluZA, D. A. Predicting the potential invasive distribuitions of four Plant species in North American. Weed Science, V. 51, P. 863 - 868. 2003.

Ramadan, Sh. E.; KHeirallah, A. M. \& AbDEl-Salam, KH. M. 2006. Marine fouling community in the Eastern harbour of Alexandria, Egypt compared with four decades of previous studies. Mediterranean Marine Science 7(2): 19-29.

ReLINI, G. 2003. II biofouling. Parte prima. Il macrofouling. Biologia Marina Mediterranea 10: 285 326 ,

Rocha, R. M.; Bonnet, N. Y. K.; Baptista, M. S. \& Beltramin, F. S. 2012. Introduced and native Phlebobranch and Stolidobranch solitary ascidians (Tunicata: Ascidiacea) around Salvador, Bahia, Brazil. Zoologia 29(1): 39-53.

Rodriguez, S. R.; OJedA, F. P. \& INESTROSA, N. C. 1993. Settlement of benthic marine invertebrates. Marine Ecology Progress Series 97: 193-207.

Ruiz, G. M.; FofOnOFF, P.; CARlton, J. T.; WonhaM, M.J. \& Hines, A.H. 2000a. Invasion of coastal marine communities in North America: apparent patterns, processes, and biases. Annual Review in Ecology and Systematics 31: 481-531. 
Ruiz, G. M.; Rawlings, T. K.; Dobbs, F. C.; Drake, L. A.; Mullady, T.; HuQ, A. \& Colwell, R. R. 2000b. Global spread of microorganisms by ships. Ballast water discharged from vessels harbours a cocktail of potential pathogens. Nature 408: 49-50.

Silva, A. K. P.; Mayal, E. M.; Mello, R. L. S. \& Fernandes, M. L. B. 2001. Estudo preliminar da bioincrustação sobre substratos naturais, como indicador de impacto na região do Complexo Portuário de Suape, PE. Tropical Oceanography 29(2): 139-146.

SKERMAN, T. M. 1960. Ship-fouling in New Zealand Waters: a survey of marine fouling organisms from vessels of the coastal and overseas trades. New Zealand Journal of Science 3(4): 620-648.

SluYs, R.; FAubel, A.; RAJAGOPAL, S. \& VAN DER Velde, G. 2005. A new and alien species of " oyster leech" (Platyhelminthes, Polycladida, Stylochidae) from the brackish North Sea Canal, The Netherlands. Helgoland Marine Research 59(4): 310-314.

Somerfield, P. J., FonsêCa-Genevois, V. G., Rodrigues, A. C. L., Castro, F. J. V. \& Santos, G. A. P. 2003. Factors affecting meiofaunal community structure in the Pina Basin, an urbanized embayment on the coast of Pernambuco, Brazil. Journal of the Marine Biological Association of the United Kingdom 83(6): 1209-1213.

Sutherland, J. P. 1974. Multiple stable points in natural communities. American Naturalist 108(964): 859-873.

TOMMASI, L. R.; BAUER, L. A. F.; LARA, G. A. 1972. Controle do desenvolvimento de incrustações biológicas em tubulações de Fundações de ponte marítima, em sistema de concretagem submersa "prepakt". Boletim Instituto Oceanográfico. , 21:117-128

Xavier, E. A.; Gama, B. A. P.; Porto, T. F.; Antunes, B. L. \& Pereira, R. C. 2008. Effects of disturbance area on fouling communities from a tropical environment: Guanabara Bay, Rio de Janeiro, Brazil. Brazilian Journal of Oceanography 56(2): 73-84

WEISS, C. M. 1947. The comparative tolerances of some fouling organisms to cooper and mercury. The Biological Bulletin 93(1): 56-63.

WHOI. 1952. Marine fouling and its prevention. Maryland, Woods Hole Oceanographic InstituteWHOI. 388 p.

Williams, R. J.; GRIFFITHS, F. B.; VAN DER WAL, E. J. \& KeLLY, J. 1988. Cargo vessel ballast water as a vector for the transport of nonindigenous marine species. Estuarine, Coastal and Shelf Science 26(4): 409-420.

ZvYaginTSEV, A. Y. 2003.Introduction of species into the Northwestern Sea of Japan and the problem of marine fouling. Russian Journal of Marine Biology 29(1): S10-S21. 